cm071359q: Supporting Information for

\title{
Cholesterol Phenoxy Hexanoate Mesogens: Effect of meta Substituents on Their Liquid Crystalline Behavior and In Situ Metal Nanoparticle Synthesis
}

\author{
Praveen Kumar Vemula ${ }^{\dagger}$, V. Ajay Mallia ${ }^{\dagger}$, Kujtim Bizati, George John ${ }^{*}$ \\ Department of Chemistry, The City College of New York, and The Graduate School \\ and University Center of The City University of New York, New York, NY 10031, \\ Ph. +1-212-650-8353, Fax: +1-212-650-6107. Correspondence should be addressed \\ to G. J. (john@sci.ccny.cuny.edu). \\ ${ }^{\dagger}$ Authors contributed equally.
}

Table of Contents:

Materials and methods

Supplementary Scheme 1

Synthesis procedures and characterization

Supplementary Figure S1

Supplementary Figure S2

Supplementary Figures S3-S6

Supplementary Figure S7 pages: S2

Synthetic scheme for mesogens, page S3

pages: S3-S5

Layered arrangement of mesogens

Page: S5

photo of LC and LC-GNPs film.

page: S6

OPM images of LCs and LC-GNPs

pages: S7-S10

Images of histogram analysis 


\section{Materials and Methods}

The high-resolution proton NMR spectra of all the intermediates and the final compounds have been recorded in a Varian (300 MHz) spectrometer using $\mathrm{CDCl}_{3}$ as the solvent. The optical textures of the mesophases were observed and identified using the polarizing optical microscope (Leica DMLB2) equipped with the hot stage (Mettler FP82). The phases obtained under polarizing microscope were also confirmed using differential scanning calorimeter (Mettler DSC-822). All measurements were conducted at the heating/cooling rate of $5{ }^{\circ} \mathrm{C}$.

Preparation of GNPs: For the in situ preparation of gold nanoparticles, mesogen and $\mathrm{HAuCl}_{4}$ was dissolved in acetone and few drops of homogeneous solution was placed on glass slide and dried to form a thin film, then heated on a hot stage (at the specific temperature) and cooled to room temperature. The reduced sample were contained GNPs dissolved in acetone and a drop was placed on Cu-grid, after drying at ambient conditions examined under transmission electron microscope. 
Scheme S1. Chemical structures and synthetic scheme of mesogens. ${ }^{a}$



cholesterol

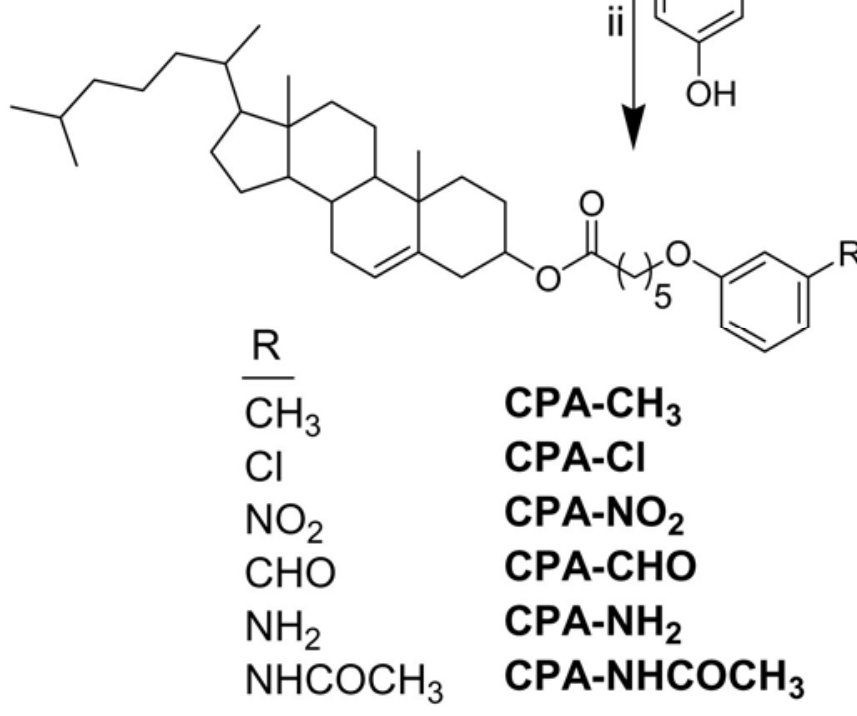

${ }^{a}$ Reagents and conditions. (i) 6-bromo hexanoic acid, dicyclohexyl carbodiimide (DCC), 4-dimethylaminopyridine (DMAP), dichloromethane, rt, 24 hrs. (ii) $\mathrm{K}_{2} \mathrm{CO}_{3}$, DMF, 100 ${ }^{\circ} \mathrm{C}, 40$ hrs.

\section{Synthesis of Mesogens}

Cholesterol esters. Cholesterol was dissolved in dry dichloromethane to that 1.1 equivalent of dicyclohexyl carbodiimide (DCC), catalytic amount of dimethylaminopyridine (DMAP) and 1.2 equivalents of 6-bromohexanoic acid were added and stirred at room temperature for $24 \mathrm{hr}$. After the reaction time, dicyclohexyl urea (DCU) was filtered and washed with dichloromethane, and the filtrate was washed 
with slightly acidic solution and extracted with dichloromethane. Organic layer was dried over anhydrous $\mathrm{Na}_{2} \mathrm{SO}_{4}$. Crude material was subjected to silica-gel column chromatography for purification.

Cholesteryl phenoxy alkanoates. The cholesteryl ester which was obtained from above mentioned procedure was dissolved in dry dimethylformamide (DMF) and to that anhydrous $\mathrm{K}_{2} \mathrm{CO}_{3}$ and $m$-substituted phenol was added and heated at $100{ }^{\circ} \mathrm{C}$ for $40 \mathrm{hr}$. After removal of DMF, the reaction mixture was subjected to silica-gel column chromatography to obtain final compounds as pure solids.

\section{Characterization details of CPA derivatives}

Cholesteryl[6(3-methylphenoxy)]hexanoate, $\mathbf{C P A}-\mathbf{C H}_{3}$ : Eluent: Ethyl acetate: hexane (1:9): Yield 60 \%: ${ }^{1} \mathrm{H}-\mathrm{NMR}\left(\mathrm{CDCl}_{3}, 300\right.$ (MHz): $\delta$ 0.67-1.98 (m) 2.31 (2H, t), $3.92(2 \mathrm{H}$, t), $4.62(1 \mathrm{H}, \mathrm{m}), 5.37(1 \mathrm{H}, \mathrm{m}), 6.7(3 \mathrm{H}, \mathrm{m}), 7.1(1 \mathrm{H}, \mathrm{t})$.

Cholesteryl[6(3-chlorophenoxy)]hexanoate, CPA-Cl: Eluent: Ethyl acetate: hexane (1:9): Yield 68 \%: ${ }^{1} \mathrm{H}-\mathrm{NMR}\left(\mathrm{CDCl}_{3}, 300\right.$ (MHz): $\delta$ 0.67-1.98 (m) 2.29 (2H, t), 3.93 (2H, t), $4.62(1 \mathrm{H}, \mathrm{m}), 5.37$ (1H, m), $6.75(1 \mathrm{H}, \mathrm{d}), 6.90(2 \mathrm{H}, \mathrm{m}), 7.1(1 \mathrm{H}, \mathrm{t})$.

Cholesteryl[6(3-nitrophenoxy)]hexanoate, CPA-NO ${ }_{2}$ : Eluent: Chloroform: Yield 90 \%: ${ }^{1} \mathrm{H}-\mathrm{NMR}\left(\mathrm{CDCl}_{3}, 300(\mathrm{MHz}): \delta\right.$ 0.67-1.98 (m) $2.31(2 \mathrm{H}, \mathrm{t}), 4.0(2 \mathrm{H}, \mathrm{t}), 4.62(1 \mathrm{H}, \mathrm{m})$, $5.37(1 \mathrm{H}, \mathrm{m}), 7.20(1 \mathrm{H}, \mathrm{d}), 7.39(1 \mathrm{H}, \mathrm{t}), 7.7(1 \mathrm{H}, \mathrm{m}), 7.8(1 \mathrm{H}, \mathrm{d})$.

Cholesteryl[6(3-formylphenoxy)]hexanoate, CPA-CHO: Eluent: Chloroform: Yield 80 \%: ${ }^{1} \mathrm{H}-\mathrm{NMR}\left(\mathrm{CDCl}_{3}, 300(\mathrm{MHz}): \delta\right.$ 0.67-1.98 (m) $2.31(2 \mathrm{H}, \mathrm{t}), 4.0(2 \mathrm{H}, \mathrm{t}), 4.62(1 \mathrm{H}, \mathrm{m})$, $5.37(1 \mathrm{H}, \mathrm{m}), 7.14(1 \mathrm{H}, \mathrm{m}), 7.36(1 \mathrm{H}, \mathrm{m}), 7.4(2 \mathrm{H}, \mathrm{m}), 9.96(1 \mathrm{H}, \mathrm{s})$. 
Cholesteryl[6(3-aminophenoxy)]hexanoate, $\mathrm{CPA}-\mathrm{NH}_{2}$ : Eluent: Methanol: Chloroform (1:24): Yield $60 \%:{ }^{1} \mathrm{H}-\mathrm{NMR}\left(\mathrm{CDCl}_{3}, 300(\mathrm{MHz}): \delta\right.$ 0.67-1.98 (m) $2.29(2 \mathrm{H}, \mathrm{t}), 3.63(2 \mathrm{H}$, s), $3.90(2 \mathrm{H}, \mathrm{t}), 4.62(1 \mathrm{H}, \mathrm{m}), 5.37(1 \mathrm{H}, \mathrm{m}), 6.44(3 \mathrm{H}, \mathrm{m}), 7.10(1 \mathrm{H}, \mathrm{t})$.

Cholesteryl[6(3-acetaamidophenoxy)]hexanoate, CPA-NHCOCH :

Eluent: Chloroform: Yield $69 \%$ : ${ }^{1} \mathrm{H}-\mathrm{NMR}\left(\mathrm{CDCl}_{3}, 300\right.$ (MHz): $\delta$ 0.67-1.98 (m) 2.29 (2H, t), $3.93(2 \mathrm{H}, \mathrm{t}), 4.58(1 \mathrm{H}, \mathrm{m}), 5.37(1 \mathrm{H}, \mathrm{m}), 6.62(1 \mathrm{H}, \mathrm{t}), 6.94(1 \mathrm{H}, \mathrm{t}), 7.16(2 \mathrm{H}, \mathrm{m})$.
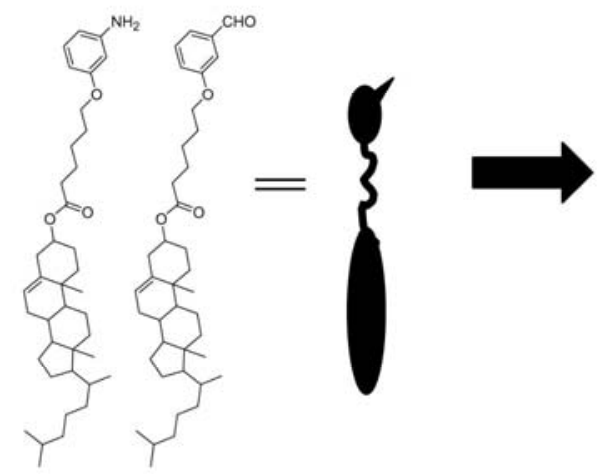



monolayer arrangement

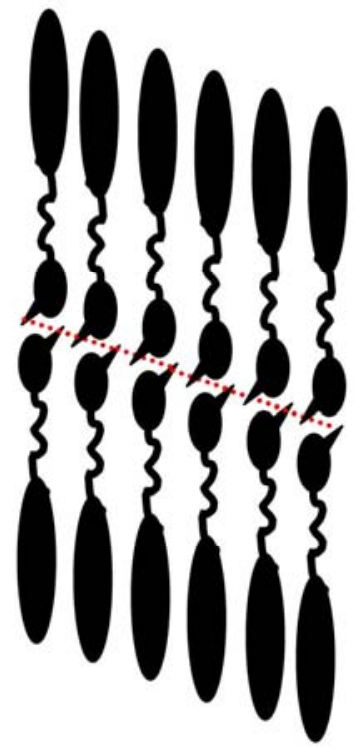

bilayer arrangement

Figure S1. Schematic representation for hypothetical layered structure arrangements of mesogens which can form lateral hydrogen bonding (H-bonds have shown in red dotted lines). 

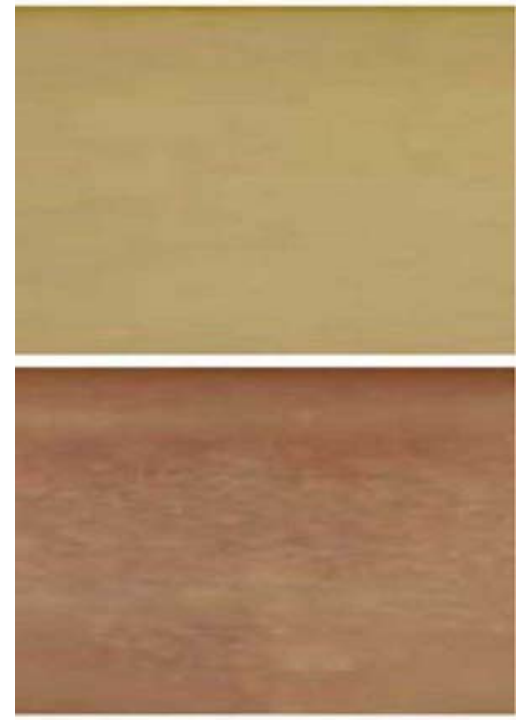

Figure S2. Photos of liquid crystal film on glass slide (top) without, and (bottom) with gold nanoparticles after reduction. 

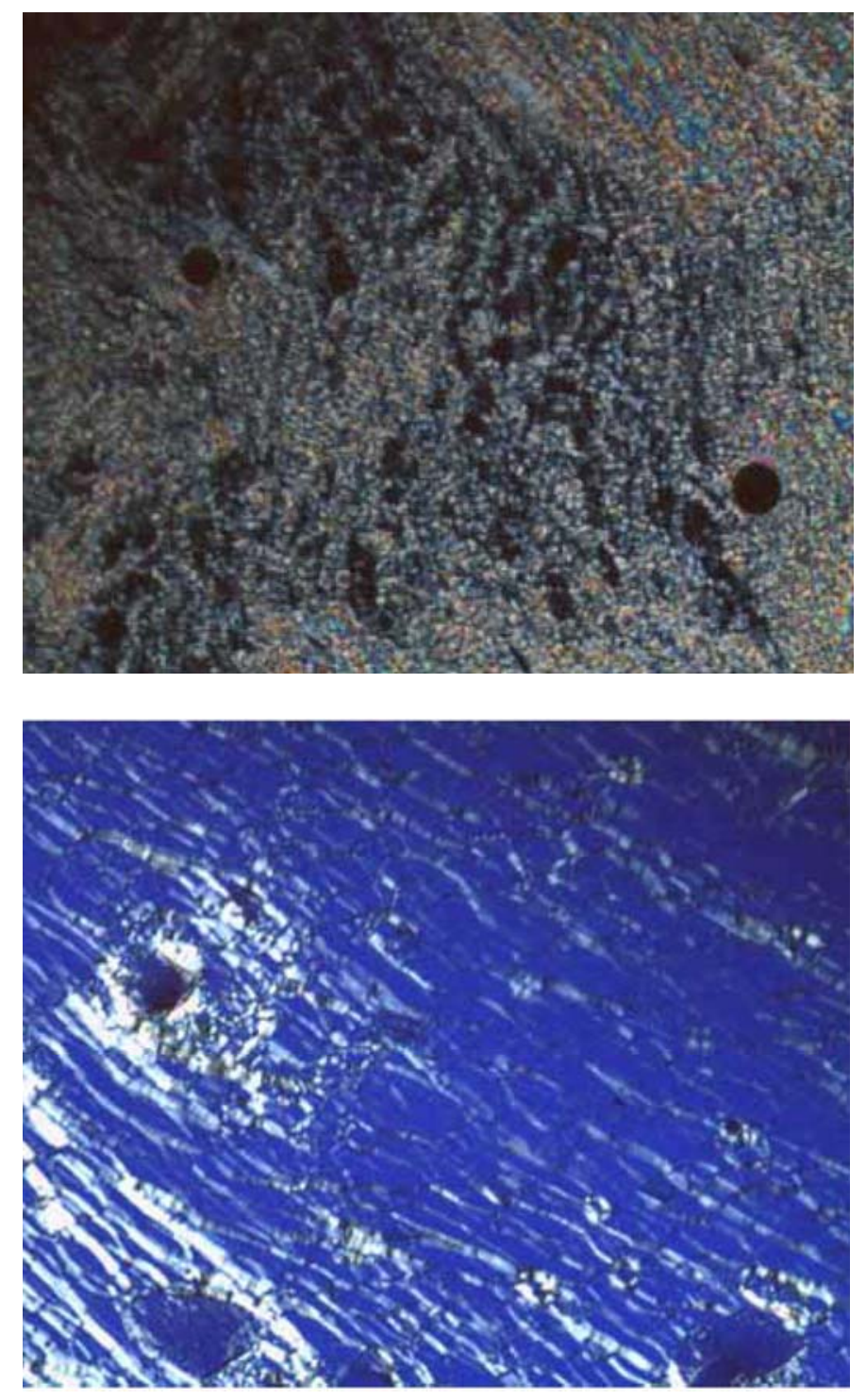

Figure S3. OPM (optical polarized microscope) images of LC phases formed from CPACHO (top) SmA* at $31^{\circ} \mathrm{C}$ and (bottom) $\mathrm{N}^{*}$ at $61^{\circ} \mathrm{C}$ in cooling cycle. 

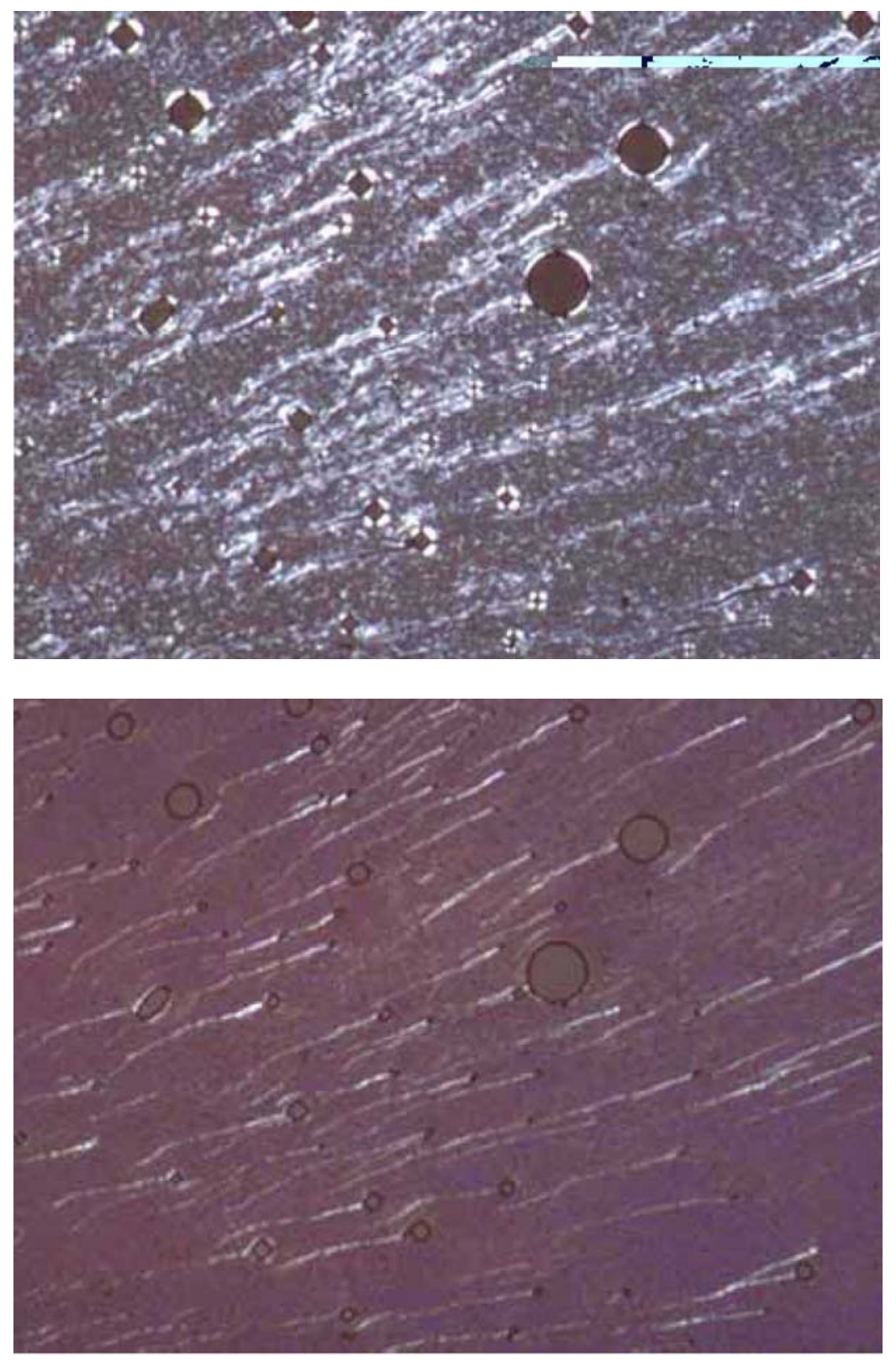

Figure S4. OPM images of LC phases formed from 5 mol\% GNPs embedded CPACHO (top) SmA* at $37^{\circ} \mathrm{C}$ and (bottom) $\mathrm{N}^{*}$ at $50^{\circ} \mathrm{C}$ in cooling cycle. 

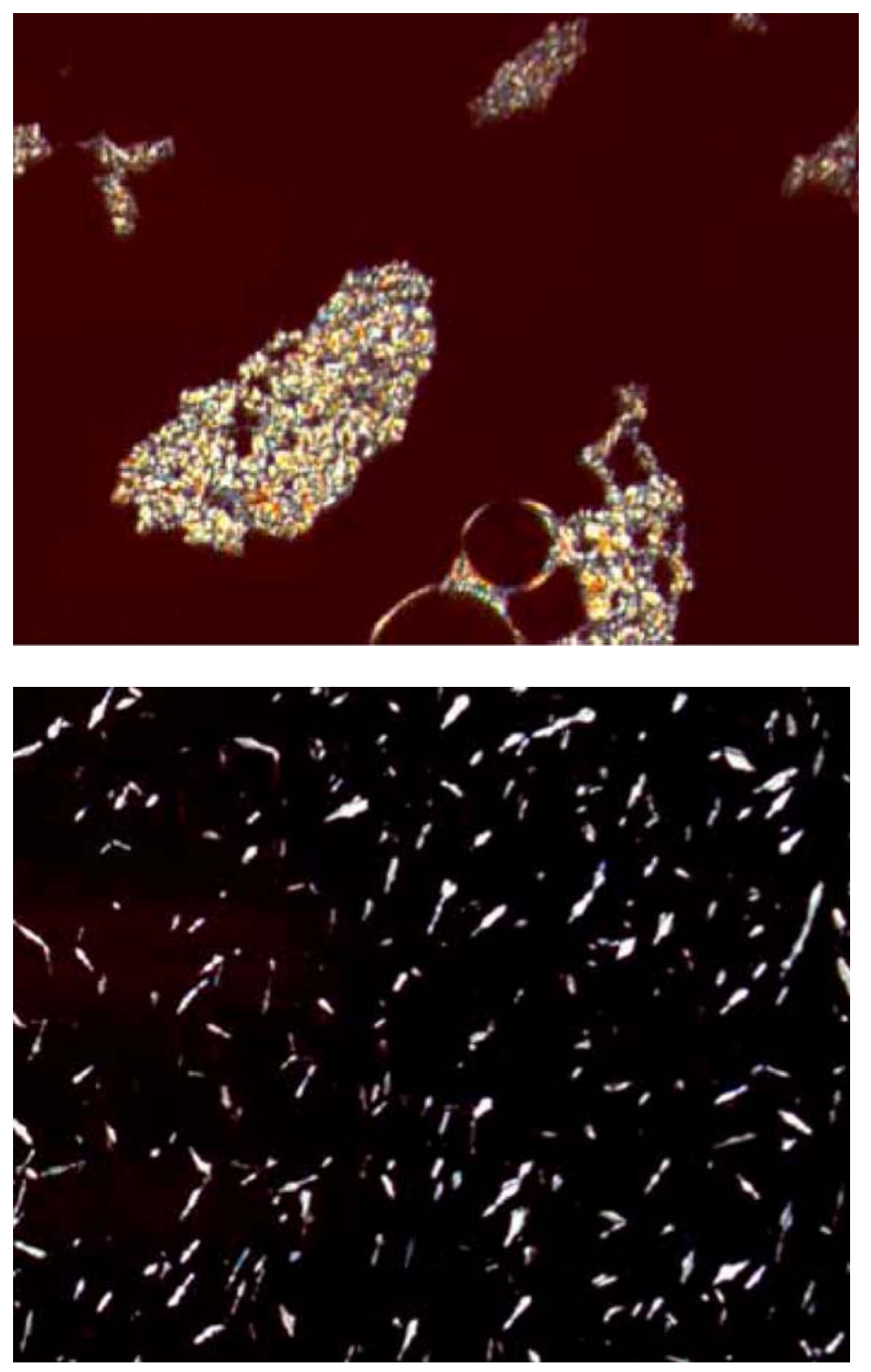

Figure S5. OPM images of SmA* phase formed from CPA-NH2 (top) without GNPs at $37^{\circ} \mathrm{C}$ and (bottom) with GNPs at $72{ }^{\circ} \mathrm{C}$ in cooling cycle. 



Figure S6. OPM images of $\mathrm{N}^{*}$ phase formed from (top) $\mathbf{C P A}-\mathbf{C H}_{3}$ at $29{ }^{\circ} \mathrm{C}$ and (bottom) CPA-Cl at GNPs at $53{ }^{\circ} \mathrm{C}$ in cooling cycles. 

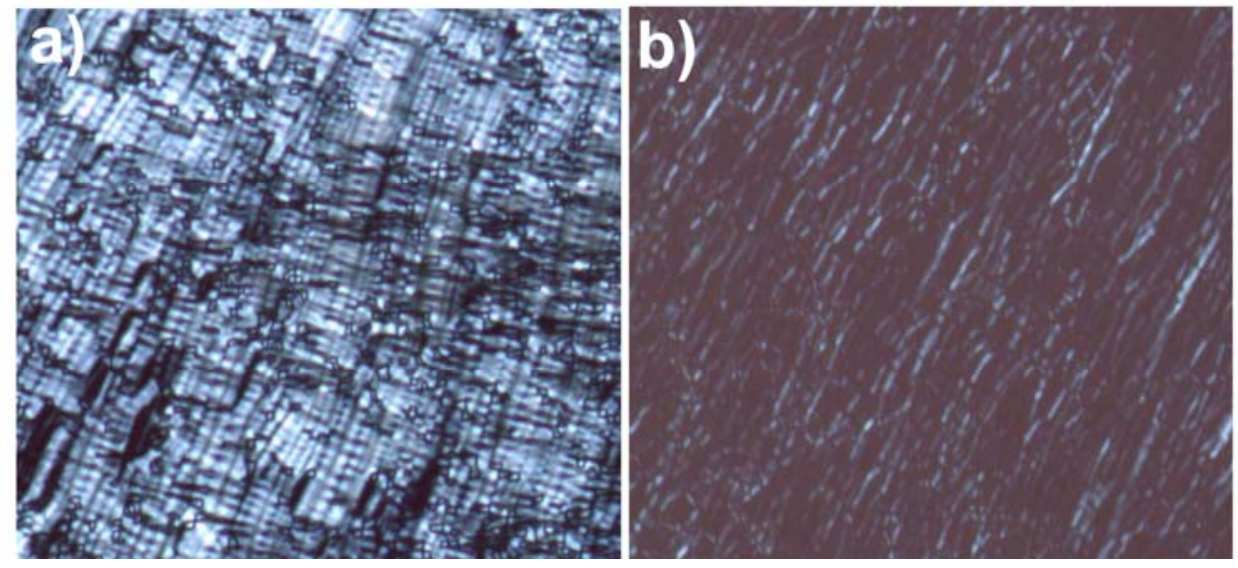

Figure S7. Optical microscope images for chiral nematic phases of (a) $\mathbf{C P A}-\mathbf{C H}_{3}$ and (b) CPA-Cl.
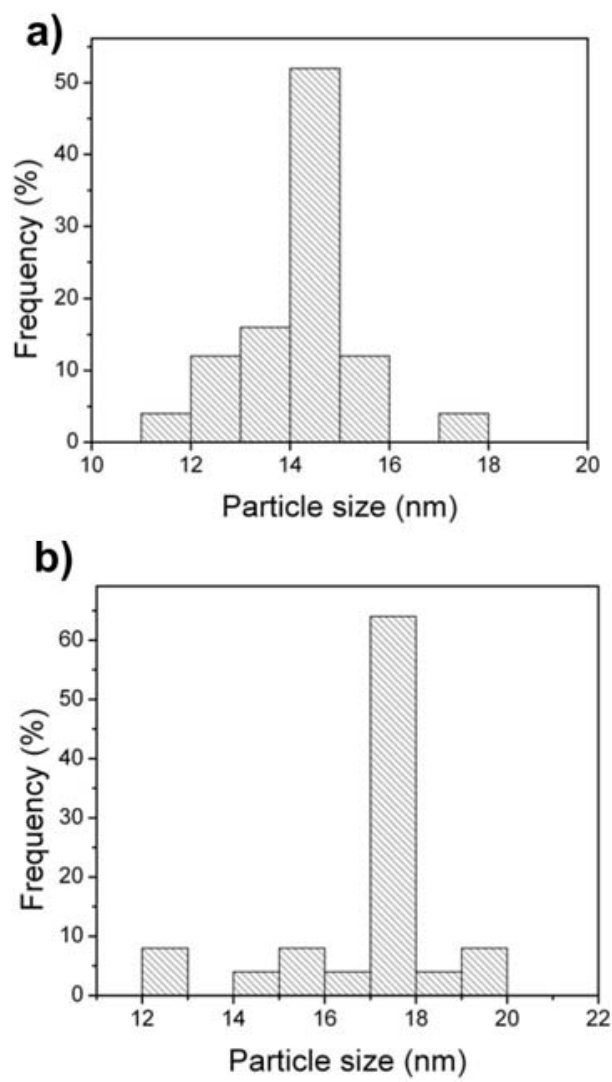

Figure S7. Histogram analysis of GNPs synthesized in a) $\mathbf{C P A}-\mathbf{N H}_{2}$ and b) CPA-CHO liquid crystal systems. 